Abstract

\title{
From Kaluza-Klein to Non-Commutative Gauge Theories of Gravity ${ }^{+}$
}

\section{George Zoupanos}

Physics Department, National Technical University of Athens, 15780 Zografou, Greece; george.zoupanos@cern.ch

† Presented at Symmetry 2017-The First International Conference on Symmetry, Barcelona, Spain, 16-18 October 2017.

Published: 9 January 2018

\begin{abstract}
Firstly; a short reminder of the Kaluza-Klein programme and a review of the Coset Space Dimensional Reduction of higher dimensional gauge theories will be given. Then; the "fuzzy sphere" will be presented; as a representative example of a non-commutative coset as well as the construction of gauge theories on such spaces. Eventually; examples will be presented of (a) a higher dimensional Unified Theory with fuzzy coset spaces as extra dimensions; (b) gravity as a gauge theory on non-commutative spaces.
\end{abstract}

(C) 2018 by the author. Licensee MDPI, Basel, Switzerland. This article is an open access article distributed under the terms and conditions of the Creative Commons Attribution (CC BY) license (http://creativecommons.org/licenses/by/4.0/). 